76 percent of beryllia $(\mathrm{BeO}), 4$ percent of alumina $\left(\mathrm{Al}_{2} \mathrm{O}_{3}\right)$, and 20 percent of zirconia $\left(\mathrm{ZrO}_{2}\right)$, with an admixture of 2 weight percent of calcia $(\mathrm{CaO})$. This body becomes practically impervious at temperatures between $1,500^{\circ}$ and $1,600^{\circ} \mathrm{C}$ in both electric and gas-fired furnaces, with a resultant shrinkage of about 20 percent and an apparent density of about 3.3 $\mathrm{g} / \mathrm{cm}^{3}$.

The results of this study indicate that some of the porcelains within the beryllia field of the system beryllia-alumina-zirconia may be of use as components for various heat engines where high strength and some degree of elasticity, rather than the ultimate in refractoriness, are desired.

The authors acknowledge the assistance of $\mathrm{L}$. G. Lambert in the preparation, maturing, and testing of the specimens.

\section{References}

[1] M. D. Burdick, R. E. Moreland, and R. F. Geller, NACA Tech. Note 1561 (April 1948).

[2] R. F. Geller and M. D. Burdick, NACA W-48 (June 1946).
[3] R, F. Geller, P. J. Yavorsky, B. L. Steirman, and A. S. Creamer, J. Research NBS 36, 277 (1946) RP1703.

[4] Ohio State University Reports (Project Ceramics, 210 252-341): No. 36-H. R. Lowers (Dec. 26, 1947); No. 39 -A. P. Welch (Jan. 15, 1948); No. 40-E. T Montgomery (March 4, 1948); and No. 44-H. R. Lowers and A. R. Blackburn (June 23, 1948).

[5] University of Illinois Report No. 26, Contract No. W33-038 ac-14520 (16071) (July 9, 1948).

[6] J. C. Freche and B. W. Sheflin, NACA Research Memor E8G20 (Sept. 1948), and J. C. Freche, NACA Research Memor. E9L07 (Feb. 1950).

[7] S. M. Lang, L. H. Maxwell, and R. F. Geller, J. Research NBS 43, 429 (1949) RP2034.

[8] R. F. Geller, J. Research NBS 27, 555 (1940) RP1443, R. F. Geller and P. J. Yavorsky, J. Research NBS 35, 87 (1943) RP1662.

[9] A. R. Bobrowsky, Conference on ceramic materials for application to aireraft power plants. Air Matériel Command, Wright-Patterson Air Force Base (May 27 to 28,1948

[10] R. A. Heindl and W. L. Pendergast, J. Am. Ceram. Soc., 10, 524 (1927).

[11] G. A. Rankin and F. E. Wright, Am. Jr. Sci., 39, 11 (1915)

[12] H. von Wartenburg, H. Reusch, and E. Saran, Z. anorg. allgem. Chem. 230, 275 (1937).

[13] R. H. Bogue, The chemistry of portland cement (Reinhold Publishing Co. Inc., New York, N. Y., 1947).

Washington, June 6, 1950.

\title{
Some Electrical Relations in Galvanic Couples
}

\author{
By H. D. Holler
}

\begin{abstract}
The electrical relations are developed for a galvanic couple (short-circuited cell) with and without polarization by externally applied current. Their significance in the derivation of criteria for cathodic protection is demonstrated. The mechanism of current flow between couples at different potentials is explained. The importance of galvanic-couple theory in governing current distribution over an electrode surface is indicated.
\end{abstract}

\section{Introduction}

In the study of electrode processes, an understanding of the electrical relations in galvanic couples and of their behavior toward externally applied current is essential. It has, of course, been known since the time of Galvani and Volta, that a bimetallic junction in contact with an electrolyte is a source of electromotive force and current. While there is some question regarding the exact source of the emf, it can be shown by thermodynamics that the chemical potential of such a system manifests itself as an emf, theoretically capable of delivering current.

In accordance with Nernst's law, it is also well known that an emf may exist between two areas of the same metal, each in different environments, and a galvanic current will flow when they are metallicly connected. Even in the case of a pure metal, in an electrolyte, galvanic currents may flow between its different crystal faces, from cracks in its surface to surrounding areas, or between areas in different

1 The term "galvanic couple" or "couple" is used in this paper for the sake of brevity and means a galvanic cell having its electrodes metallicly connected through an external resistance that may be negligibly small. states of oxidation. As a result, the conditions favoring the existence of galvanic couples in the phase boundary are nearly always fulfilled whenever a metal comes into contact with an electrolyte. In connection with the "local-cell" theory of corrosion, some of the electrical relations governing these currents have been derived by Müller, Akimow, and Thomasow, and others [1]. ${ }^{2}$ There are galvanic circuits of all magnitudes ranging from those of molecular dimensions to those of great size existing underground. They are present, to some extent, on the electrodes in practically all electrochemical processes. Systems of several electrodes have been studied by many workers including Brown and Mears, Landau and Petrocelli [2].

While thermodynamics defines the driving force of an electrochemical reaction, the rate of the latter is controlled by irreversible factors, represented by polarization, as defined in its broadest sense; that is, including resistive potential differences also. Sometimes, as in electric batteries, it is desirable that the polarization resulting from delivery of current to an

\footnotetext{
${ }^{2}$ Figures in brackets indicate the literature references at the end of this paper.
} 
external circuit be kept at a minimum. However, in these same batteries, the polarization of any galvanic couples on the surfaces of the individual electrodes should be a maximum, in order to reduce the "selfdischarge" of the electrode. This may be accomplished by the proper choice of pure materials, the use of inhibitors, or by other means.

In other electrochemical processes, such as electroplating, the reaction at the electrode may be selectively determined by its polarization. For example, when the dissolution of a metal anode with high current-efficiency is desired, additions to the electrolyte may be made for the reduction of polarization. At the cathode, the control of polarization by chemical or electrical means may determine the proportions of elements deposited and their distribution. Thus, the "throwing power" of an electroplating solution is a function of the rate of change of polarization with current [3].

In corrosion mitigation, it is fortunate when, through self-polarization of the galvanic couples, the galvanic currents become negligible. This is the basis of the mechanism of passivity developed by Mears and Brown [4]. The presence of such galvanic couples and their polarization may thereby explain why some metals do not corrode, as well as why others do corrode. The protection of a metal by a coating of another metal depends largely upon the electrical characteristics of the galvanic couples involved and the principles of cathodic protection. An understanding of these principles also requires a derivation of the relations among the electrode potentials, currents, and resistances involved in a galvanic cell on closed circuit. Since opening this circuit is usually impossible, only a few electrical measurements can be made. The most important of these is the potential of the couple with reference to another electrode of constant potential. Unfortunately, this measurement by itself is usually of limited value in ascertaining the corrosion status.
However, if an external current be applied to the couple, any change in the relation of its potential to the applied current may supply valuable information. Before such information may be correctly interpreted, an elementary analysis of the electrical relations is required.

\section{Derivation of Basic Electrical Relations}

Let figure 1, $A$ represent a galvanic couple consisting of separate electrodes where $e_{a}$ and $e_{c}$ are the polarized anode and cathode potentials, ${ }^{3}$ respectively. The term "polarized potential" means that the potential is a function of current. This results from the electrochemical effects of current at the interface. We shall assume that the anode and cathode are each free from local couples. Since the effect of time will not be considered here, $e_{a}$ and $e_{c}$ are momentary values. Then by Ohm's law

$$
\left(e_{a}-e_{c}\right)-i_{o}\left(r_{a}+r_{c}\right)=0,
$$

where $r_{a}$ and $r_{c}$ are the resistances, mainly ionic, associated with the anode and cathode, respectively, and $i_{0}$ is the momentary value of the galvanic current. The external resistances $R_{a}$ and $R_{c}$ between $e_{a}$ and $e_{c}$ are neglected here and throughout this paper, as their omission makes no difference in the argument. The potential difference $\left(E_{G}-E_{s}\right)$ between the terminal $G$ and an electrode having a constant potential $E_{s}$ may be measured with a potentiometer and expressed by the following equations:

$$
\left(E_{G}-E_{s}\right)-\left(e_{a}-E_{s}\right)+i_{o} r_{a}=0
$$

and

$$
-\left(E_{G}-E_{s}\right)+\left(e_{c}-E_{s}\right)+i_{o} r_{c}=0 .
$$

If now a potential difference $E_{t}$ be applied by closing $k_{B}$ in figure $1, \mathrm{~B}$, a current $I$ will be superposed on

3 "Potential" as used in this paper is a potential level referred to some electrode of constant value in the same electrolyte.
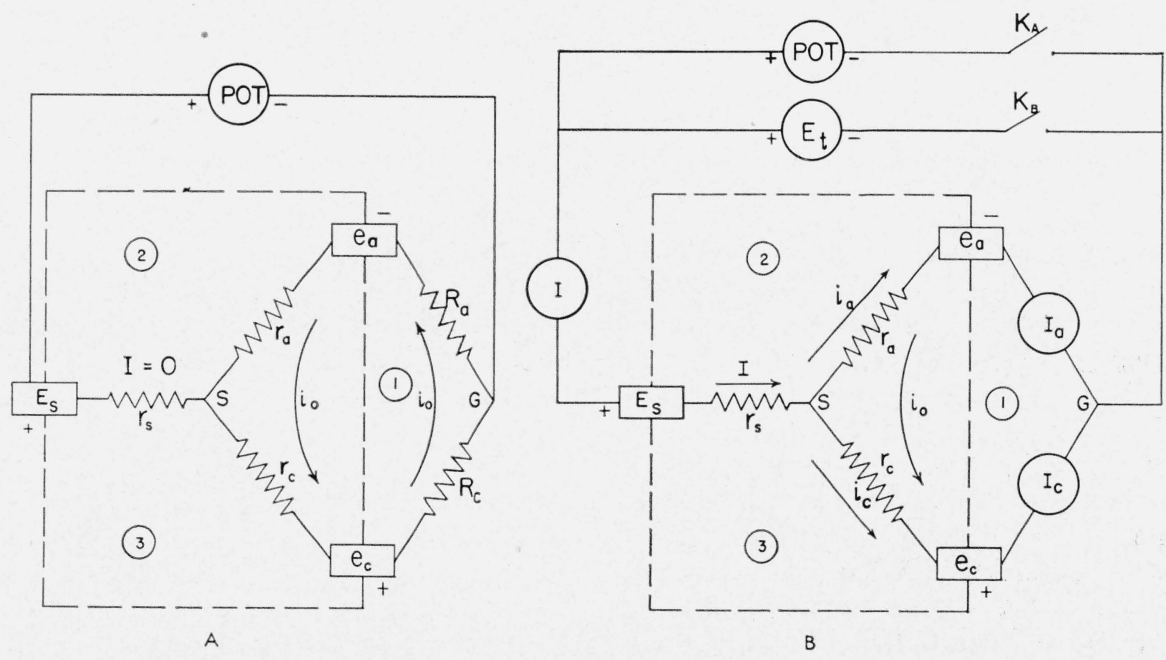

FIGURE 1. Electrical relations between a galvanic couple and reference electrode.

POT is a high-resistance voltage indicator. A, Without external current; B, with external current. 
the galvanic couple if $E_{t} \neq\left(E_{G}-E_{s}\right)$. It will flow internally from $E_{s}$ toward the couple if $E_{t}>\left(E_{G}-E_{s}\right)$. We shall consider $E_{s}$ to be unpolarized, that is, its potential is not changed by current $I$. Let $r_{s}$ be the ionic resistance between $E_{s}$ and the point $S$ where $I$ divides into two components, $i_{a}$ through $r_{a}$ and $i_{c}$ through $r_{c}$. Then

$$
I_{a}=i_{a}-i_{o} \text { and } I_{c}=i_{c}+i_{o},
$$

$I_{a}$ and $I_{c}$ being measurable.

At the instant of closing the switch $k_{B}$, we shall assume that $e_{a}$ and $e_{c}$ as well as $r_{a}$ and $r_{c}$, are unchanged by current $I$. As a result the momentary value of $i_{o}$ is the same as in figure $1, A$, and eq 1,2 , and 3 still hold. We shall consider the distribution of $I_{a}$ and $I_{c}$ such that each electrode is either wholly anodic, or wholly cathodic.

Taking meshes 1 and 2 in figure $1, B$ clockwise, then by Kirchoff's laws:

$$
\left(e_{a}-e_{c}\right)+\left(i_{a}-i_{o}\right) r_{a}-\left(i_{c}+i_{o}\right) r_{c}=0 .
$$

Likewise

$$
E_{t}-\left(e_{a}-E_{s}\right)-\left(i_{a}-i_{o}\right) r_{a}-I r_{s}=0 .
$$

Now let

$$
E_{t}-I r_{s}=E,
$$

where $E=$ that portion of the potential difference $E_{t}$ that is applied directly to the couple between the junction point $S$ and the terminal $G$.

Rewriting eq 5 and 6

$$
\begin{aligned}
\left(i_{a}-i_{o}\right) r_{a}-\left(i_{c}+i_{o}\right) r_{c} & =-\left(e_{a}-e_{c}\right), \\
-\left(i_{a}-i_{o}\right) r_{a} & =-\left[E-\left(e_{a}-E_{s}\right)\right]
\end{aligned}
$$

and solving

$$
i_{a}-i_{o}=\frac{-r_{c}\left[E-\left(e_{a}-E_{s}\right)\right]}{-r_{a} r_{c}}=\frac{E-\left(e_{a}-E_{s}\right)}{r_{a}}=I_{a} .
$$

Likewise

$$
\begin{gathered}
i_{c}+i_{o}=\frac{-r_{a}\left[E-\left(e_{a}-E_{s}\right)\right]-r_{a}\left(e_{a}-e_{c}\right)}{-r_{a} r_{c}}= \\
\frac{E-\left(e_{c}-E_{s}\right)}{r_{c}}=I_{c} .
\end{gathered}
$$

In eq 10 and 11 , it is seen that for given values of $e_{a}$ and $e_{c}$ the relative magnitude of $E$ determines the direction of the current through each electrode. For example, no current flows to the anode unless $E>\left(e_{a}-E_{s}\right)$.

Then $i_{a}>i_{o}$ and $I_{a}>0$.

Now let us derive additional equations evaluating the same currents. Again, by Kirchoff's laws, we have

$$
\begin{aligned}
& \left(i_{a}-i_{o}\right)+\left(i_{c}+i_{o}\right)=I, \\
& \left(i_{a}-i_{o}\right) r_{a}-\left(i_{c}+i_{o}\right) r_{c}=-\left(e_{a}-e_{c}\right),
\end{aligned}
$$

and solving

$i_{a}-i_{o}=\frac{-I r_{c}+\left(e_{a}-e_{c}\right)}{-r_{c}-r_{a}}=-\frac{\left(e_{a}-e_{c}\right)}{\left(r_{a}+r_{c}\right)}+\frac{I r_{c}}{\left(r_{a}+r_{c}\right)}=I_{a}$,

and

$$
i_{c}+i_{o}=\frac{-\left(e_{a}-e_{c}\right)-I r_{a}}{-r_{c}-r_{a}}=\frac{\left(e_{a}-e_{c}\right)}{\left(r_{a}+r_{c}\right)}+\frac{I r_{a}}{r_{a}+r_{c}}=I_{c} .
$$

In eq 13 and 14 it is seen that, since from eq 1

$$
\begin{gathered}
\frac{e_{a}-e_{c}}{r_{a}+r_{c}}=i_{o}, \\
i_{a}=\frac{I r_{c}}{r_{a}+r_{c}} \text { and } i_{c}=\frac{I r_{a}}{r_{a}+r_{c}} .
\end{gathered}
$$

Before switch $k_{B}$ was closed, the emf $\left(E_{G}-E_{s}\right)$ of the galvanic couple followed from eq 2 and 3 . Thus,

$$
\begin{aligned}
\left(E_{G}-E_{s}\right) & =\left(e_{a}-E_{s}\right)-i_{o} r_{a} \\
& =\left(e_{c}-E_{s}\right)+i_{o} r_{c}
\end{aligned}
$$

On closing $k_{B}$ the applied potential difference $E_{t}$ is opposed by emf, $\left(E_{G}-E_{s}\right)$.

Thus

$$
E_{t}-\left(E_{G}-E_{s}\right)-I r_{s}-I R_{G}=0,
$$

where $R_{G}$ is the resistance of the couple.

Introducing $E$, as defined in eq 7

$$
\frac{E-\left(E_{G}-E_{s}\right)}{I}=R_{G} .
$$

To evaluate $R_{G}$, let us proceed by equating the two expressions for $i_{a}-i_{o}$ given in eq 10 and 13 . Thus,

$$
i_{a}-i_{o}=\frac{E-\left(e_{a}-E_{s}\right)}{r_{a}}=\frac{I r_{c}}{\left(r_{a}+r_{c}\right)}-\frac{\left(e_{a}-e_{c}\right)}{\left(r_{a}+r_{c}\right)}=I_{a} .
$$

Since $\left(e_{a}-e_{c}\right) /\left(r_{a}+r_{c}\right)=i_{o}$, eq 20 becomes

$$
E-\left(e_{a}-E_{s}\right)=\frac{I r_{a} r_{c}}{r_{a}+r_{c}}-i_{o} r_{a},
$$

and since eq 17a may be written

$$
E_{G}=e_{a}-i_{o} r_{a}
$$

eq 21 becomes

$$
E-\left(E_{G}-E_{s}\right)=I \frac{r_{a} r_{c}}{r_{a}+r_{c}},
$$

then from eq 19 and 23 we obtain

$$
R_{G}=\frac{r_{a} r_{c}}{r_{a}+r_{c}} .
$$




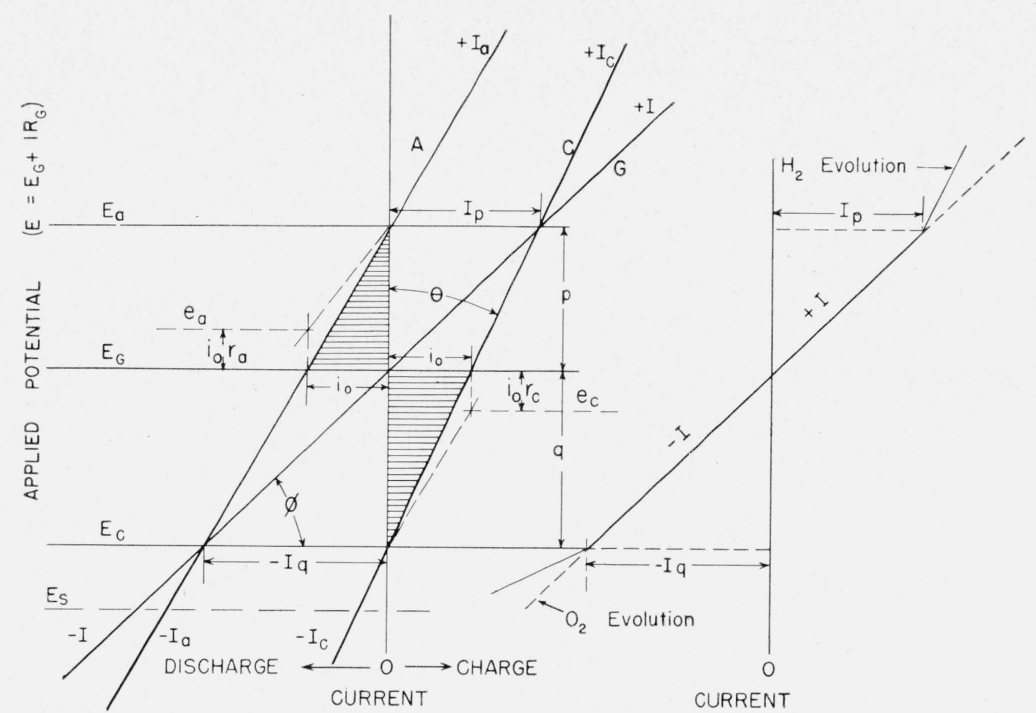

Figure 2. Polarization curves for anode A, cathode C, and galvanic couple $G$, showing possible discontinuities at $I_{p}$ and $I_{q}$.

\section{Polarization of a Galvanic Couple}

In order to understand more clearly the potentialcurrent relations resulting from the application of current to a galvanic couple, let us synthesize the diagram in figure 2, where all quantities indicated may be measured directly or calculated from experimental data. It comprises three polarization curves, one for the anode $A$, one for the cathode $C$, and one for the galvanic couple $G$. Consider two separate electrodes, $A$ and $C$, having open-circuit potentials $E_{a}$ and $E_{c}$, and a reference electrode at potential level $E_{s}$, all three being in the same electrolyte. Beginning with zero current, let us polarize the electrode potential represented by $E_{c}$, cathodically, that is, pass current $I_{c}$ from the electrolyte to the electrode. In order to do this, a potential difference $E$ greater than $E_{c}$ is required. The relation $E / I_{c}$ represents the cathode polarization curve, and when $I_{c}$ becomes equal to $I_{p}$, the cathode potential is equal to $E_{a}$. Next, beginning with zero current, let us polarize anodically the electrode whose "opencircuit" potential is represented by $E_{a}$, that is, pass current, $I_{a}$ from electrode to electrolyte. This requires a potential difference $E$ less than $E_{a}$. The relation $E /-I_{a}$ represents the anode polarization curve, and when $-I_{a}$ becomes equal to $-I_{q}$, the anode potential is equal to $E_{c}$.

After these polarization curves have been separately constructed, let the two electrodes be electrically connected. Then when the potential, $E$, is equal to $E_{G}$, no external current $I$ enters or leaves the couple, and $-I_{a}=I_{c}=i_{o}$. If now we polarize the couple $G$ cathodically by increasing $I$ in the "charge" direction the applied potential required is $E=E_{G}+I R_{G}$ where $R_{G}$ is the resistance of the couple.

When $E=E_{G}, I=0$; when $E=E_{a}, I_{a}=0$, and when $E=E_{c}, I_{c}=0$. The conditions for cathodic protec- tion are $I_{a}=0, E=E_{a}$ and $I=I_{c}=I_{p}=$ the protective current.

Then, from eq 13 we have

$$
\frac{e_{a}-e_{c}}{r_{a}+r_{c}}=I p \frac{r_{c}}{r_{a}+r_{c}}
$$

likewise, when $I_{c}=0,-I=I_{a}=I_{q}$, and from eq 14 we have

$$
-\frac{e_{a}-e_{c}}{r_{a}+r_{c}}=I q \frac{r_{a}}{r_{a}+r_{c}}
$$

then

$$
\frac{e_{a}-e_{c}}{r_{c}}=I p \text { and } \frac{e_{a}-e_{c}}{r_{a}}=I q
$$

and

$$
\frac{I_{p} \cdot I_{q}}{I_{p}+I_{q}}=\frac{e_{a}-e_{c}}{r_{a}+r_{c}}=i_{o}
$$

It also follows from eq 27 that

$$
\frac{I_{p}}{I_{q}}=\frac{r_{a}}{r_{c}} .
$$

Equation 28 may also be derived from the trigonometry of figure 2 as follows:

$$
\begin{gathered}
\tan \theta=\frac{i_{o}}{q}=\frac{I_{p}}{p+q} \\
\tan \phi=\frac{q}{I_{q}}=\frac{p+q}{I_{p}+I_{q}} .
\end{gathered}
$$

Rearranging and combining

$$
i_{o}=\frac{I_{p} \cdot q}{p+q}=\frac{I_{p} \cdot I_{q}}{I_{p}+I_{q}} .
$$


If, therefore, it be possible to estimate $I_{p}$ and $I_{q}$ from changes in the $E / I$ relation as indicated in figure 2 , the magnitude of galvanic current $i_{0}$ may be evaluated from eq 28 and 32 ; and the ratio of the electrode resistances $r_{a} / r_{c}$ may be obtained from eq 29. The measurement of $I_{q}$, and particularly $I_{p}$, by any means, direct or indirect, is therefore of prime importance in evaluating the characteristics of a galvanic couple.

\section{Partition of the Applied Current}

The partition of $I$ into its anodic and cathodic components may be interpreted either from eq 10 and 11 or from eq 13 and 14 , and figure $1 B$.

Since

$$
\frac{i_{a}-i_{o}}{i_{c}+i_{o}}=\frac{I_{a}}{I_{c}}
$$

the term "partition" is ambiguous unless defined as $i_{a} / i_{c}$ or $I_{a} / I_{c}$. If the measurable ratio be used, then

or

$$
\frac{I_{a}}{I_{c}}=\frac{\left[E-\left(e_{a}-E_{s}\right)\right] \cdot r_{c}}{\left[E-\left(e_{c}-E_{s}\right)\right] \cdot r_{a}}
$$

$$
\frac{I_{a}}{I_{c}}=\frac{\frac{e_{a}-e_{c}}{r_{a}+r_{c}}+\frac{I r_{c}}{r_{a}+r_{c}}}{\frac{e_{a}-e_{c}}{r_{a}+r_{c}}+\frac{I r_{a}}{r_{a}+r_{c}}}=\frac{-i_{o}+\frac{I r_{c}}{r_{a}+r_{c}}}{i_{o}+\frac{I r_{a}}{r_{a}+r_{c}}} .
$$

If $e_{a}=\epsilon_{c}, i_{o}=0$, and both eq 34 and 35 become

$$
\frac{I_{a}}{I_{c}}=\frac{r_{c}}{r_{a}} \text {. }
$$

When $\epsilon_{a}$ and $e_{c}$ are not equal, partition of current $I_{a} / I_{c}$ is determined by the product of the emfdifference ratio $E-\left(e_{a}-E_{s}\right) / E-\left(e_{c}-E_{s}\right)$ and the resistance ratio $r_{c} / r_{a}$ as in eq 34 .

The partition of current may also be expressed by an equation, including a term that expresses the rate of change of polarization of the anode with current; and also a similar one for the cathode.

Thus, from eq 10 and 11 , and still assuming $E_{s}$ to be constant, we have by elimination of $E$,

$$
\frac{I_{a}}{I_{c}}=\frac{r_{c}+\frac{\left(e_{c}-E_{s}\right)}{I_{c}}}{r_{a}+\frac{\left(e_{a}-E_{s}\right)}{I_{a}}} .
$$

Taking the partial derivative of eq 10 with respect to $I_{a}$, we have

$$
\frac{\partial E}{\partial I_{a}}=r_{a}+\frac{\partial}{\partial I_{a}}\left(e_{a}-E_{s}\right), r_{a} \text { constant }
$$

similarly from eq 11 we have

$$
\frac{\partial E}{\partial I_{c}}=r_{c}+\frac{\partial}{\partial I_{c}}\left(e_{c}-E_{s}\right), r_{c} \text { constant }
$$

and dividing $37 \mathrm{~b}$ by $37 \mathrm{a}$ we have

$$
\left.\frac{\partial E}{\partial I_{c}}\right|_{\frac{\partial E}{\partial I_{a}}}=\frac{\partial I_{a}}{\partial I_{c}}=\frac{r_{c}+\frac{\partial}{\partial I_{c}}\left(e_{c}-E_{s}\right)}{r_{a}+\frac{\partial}{\partial I_{a}}\left(e_{a}+E_{s}\right)},
$$

where $\partial\left(e_{c}-E_{s}\right) / \partial I_{c}$ and $\partial\left(e_{a}-E_{s}\right) / \partial I_{a}$ represent the rates of change in counter emfs $e_{c}$ and $e_{a}$ with currents, $I_{c}$ and $I_{a}$ respectively.

Equation 37 is a general expression of the potentialcurrent relations, which govern the current distribution over any electrode, For example, in electroplating, the current is opposed not only by resistance but also by the emf of polarization, which tends to divert the current to more remote areas and thereby exhibit "throwing power". Also, in cathodic protection a high rate of change of counter emf with current tends to divert more current to remote areas than if current partition were controlled entirely by resistance,

\section{Measurement of $E_{G}$}

As previously indicated, the chief measurable characteristic of a "closed circuit" galvanic cell is its potential $E_{G}$ with reference to a standard electrode $E_{s}$.

Equations 17 represent the value of the emf $\left(E_{G}-E_{s}\right)$, which is indicated graphically in figure 3, $A$ and $B$. When an external emf $E_{t}$ is applied, $\left(k_{B}\right.$ closed, fig. $1 B$ ) and $E>\left(E_{G}-E_{s}\right)$, the resistive components indicated in eq 38 are added to eq 17 , giving eq 39.

$$
\begin{aligned}
I R_{G} & =i_{a} r_{a}=i_{c} r_{c} \\
\left(E_{G}-E_{s}\right)+I R_{G} & =\left(e_{a}-E_{s}\right)-i_{o} r_{a}+i_{a} r_{a} \\
& =\left(e_{c}-E_{s}\right)+i_{o} r_{c}+i_{c} r_{c}
\end{aligned}
$$

or, using the real currents, $I_{a}$ and $I_{c}$,

$$
\begin{aligned}
\left(E_{G}-E_{s}\right)+I R_{G} & =\left(e_{a}-E_{s}\right) \pm I_{a} r_{a} \\
& =\left(e_{c}-E_{s}\right)+I_{c} r_{c}
\end{aligned}
$$

$I_{a}$ is positive if $E>\left(e_{a}-E_{s}\right)$ and negative if $E<$ $\left(e_{a}-E_{s}\right)$ as in figure $3 B$. With current $I$ flowing $\left(k_{A}\right.$ and $k_{B}$ closed) through the couple, the potential difference $E_{t}=\left(E_{G}-E_{s}\right)+I r_{s}+I R_{G}$ is measured directly, by using a potentiometer.

In order to determine $\left(E_{G}-E_{s}\right)$ during passage of external current $I$, it is necessary to cancel the resistive component $I r_{s}+I R_{G}$ from the above potentiometer measurement. Two methods of doing this are briefly as follows: first, if $k_{A}$, figure $1, B$, be closed immediately after opening $k_{B}, E_{G}-E_{s}$ may be observed by a suitable voltage indicator, POT. This of course may be done manually if $E_{G}$ is the same whether $I$ is on or off. However, if $E_{G}$ is ap

preciably affected by $I$, then it must be observed a 


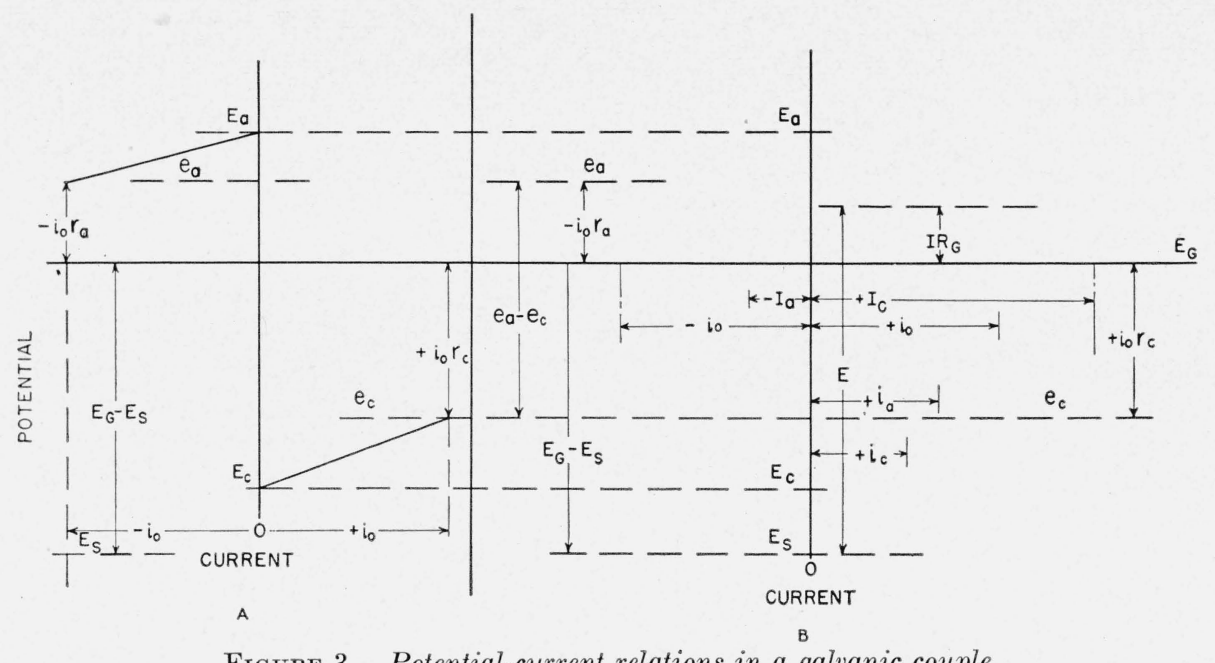

Figure 3. Potential-current relations in a galvanic couple.

$A$, Without external current; B, with external current.

the instant of current interruption. This may be done by a suitable commutator [5], which opens $k_{B}$ for short periods in succession, during which $k_{A}$ is momentarily closed, The voltage indicator will then give an average value of $E_{G}$ during the periods when $I=0$. By using the proper commutator speed, period of interruption, and indicator, the observed average can be made approximately equal to the true value of $E_{G}$ during the flow of current $I$. The use of an electronic interruptor [6] and amplifier with high-speed recorder or oscillograph [7], permits the measurement of emf, free of ir drop, with high precision.

A second method of cancelling the ir components, $I r_{s}+I R_{G}$ from the measurement of $\left(E_{G}-E_{s}\right)$ is based on the Wheatstone bridge principle [8]. To balance the bridge, having an emf in one arm, use is made of a short interruption of current $I$, or a part of $I$, by touching a key. After the balance, the values of $\left(E_{G}-E_{s}\right)$ and also of $\left(r_{s}+R_{G}\right)$ are directly observed.

Let us now suppose that through electrochemical effects resulting from flow of current, the emf, $\left(e_{a}-e_{c}\right)$ be changed to $\left(e_{a}^{\prime}-e_{c}^{\prime}\right)$. Then the galvanic current will also be changed from $i_{o}$ to $i_{o}$ in accordance with Ohm's law. Thus

$$
i_{o}^{\prime}=\frac{e_{a}^{\prime}-e_{c}^{\prime}}{r_{a}+r_{c}} .
$$

From the new values in eq 41, a new value for the galvanic couple potential is also obtained. Thus, eq 2 and 3 are written

$$
\begin{aligned}
\left(E_{G}^{\prime}-E_{s}\right) & =\left(e_{a}^{\prime}-E_{s}\right)-i_{o}^{\prime} r_{a} \\
& =\left(e_{c}^{\prime}-E_{s}\right)+i_{o}^{\prime} r_{c}
\end{aligned}
$$

The emf $\left(E_{G}^{\prime}-E_{s}\right)$ may be observed by either of the above methods. For each increment in applied current new sets of values for $i_{o}^{\prime}, e_{a}^{\prime}, e_{c}^{\prime}$ and $E_{G}^{\prime}$ are obtained, depending upon the length of time of application.
Their substitution in eq 10,11 and 13,14 give the following:

$$
\begin{aligned}
& i_{a}^{\prime}-i_{o}^{\prime}=\frac{E-\left(e_{a}^{\prime}-E_{s}\right)}{r_{a}}=I_{a}^{\prime}, \\
& i_{c}^{\prime}+i_{o}^{\prime}=\frac{E-\left(e_{c}^{\prime}-E_{s}\right)}{r_{c}}=I_{c}^{\prime}, \\
& i_{a}^{\prime}-i_{o}^{\prime}=\frac{e_{a}^{\prime}-e_{c}^{\prime}}{r_{a}+r_{c}}+I^{\prime} \frac{r_{c}}{r_{a}+r_{c}}=I_{a}^{\prime}, \\
& i_{c}^{\prime}+i_{o}^{\prime}=\frac{e_{a}^{\prime}-e_{c}^{\prime}}{r_{a}+r_{c}}+I^{\prime} \frac{r_{a}}{r_{a}-r_{c}}=I_{c}^{\prime} .
\end{aligned}
$$

Each new set of values of $I_{a}^{\prime}$ and $I_{c}^{\prime}$, if plotted according to figure 2 will give a succession of corresponding diagrams, each with a new $E_{G}^{\prime}$ level.

\section{Conditions for Cathodic Protection}

From eq $43 \mathrm{a}$ and $44 \mathrm{a}$, it follows that when $i^{\prime}{ }_{a}=i^{\prime}{ }_{o}$ or $E=\left(e^{\prime}{ }_{a}-E_{s}\right)$,
$I^{\prime}{ }_{a}=0$,

which is the "current" criterion for cathodic protection. Then also $e^{\prime}{ }_{a}=E_{a}$, and therefore $E=\left(E_{a}-\right.$ $E_{s}$ ), which is the "potential" criterion for cathodic protection.

The diagram used in figure 2 for expressing the electrical characteristics of a galvanic couple has the practical advantage that it may be constructed from results of polarization measurements on individual electrodes. However, in the case of a galvanic cell on closed circuit, the only measurable emf component is $\left(E_{G}^{\prime}-E_{s}\right)$. Let us consider what relation it bears to the criteria for cathodic protection. From eq 40a, we have

$$
\left(E_{G}^{i}-E_{s}\right)+I^{\prime} R_{G}=\left(e^{\prime}{ }_{a}-E_{s}\right) \pm I^{\prime}{ }_{a} r_{a} .
$$


When cathodic protection is achieved,

$$
\left(E^{\prime}{ }_{G}-E_{s}\right)+I^{\prime} R_{G}=\left(E_{a}-E_{s}\right) .
$$

If the measured value of $\left(E^{\prime}{ }_{G}-E_{s}\right)$ becomes equal to $\left(E_{a}-E_{s}\right)$, the applied potential exceeds the criterion for cathodic protection by the amount $I^{\prime} R_{G}$. A method of measuring $R_{G}$ (independently of $I r_{s}$ ) is not yet available. If $R_{G}$ is negligibly small, the equality $\left(E_{a}-E_{s}\right)=\left(E_{G}-E_{s}\right)$ is an accurate criterion of cathodic protection. If $R_{G}$ is relatively high, the observed value of $\left(E_{G}-E_{s}\right)$ is not an accurate criterion. This fact indicates the need for a technique by which $R_{G}$ may be measured.

The relation of $E$ to $I$ (fig. 2) will now be considered. First, if $E$ be increased from $E=E_{G}$ to $E=E_{a}$, the liberation of hydrogen may be expected to increase in proportion to the increase in $I_{c}$. If $E$ be further increased, $I_{a}>0$ and liberation of hydrogen may then be expected to increase in proportion to the increase in $\left(I_{a}+I_{c}\right)$. If $I_{a}$ is sufficiently large, the increased rate of evolution of hydrogen above $I_{p}$ may manifest itself as a discontinuity in the $E / I$ relation. If, instead of hydrogen evolution, some other reduction process occurs, such as reduction of oxygen, of a film, or of some other depolarizer, there may be no evidence of a discontinuity in the $E / I$ relation.

Second, if $E$ be decreased from $E=E_{G}$ to $E=E_{c}$, the liberation of hydrogen should decrease in proportion to the decrease in $I_{c}$. When $I$ becomes equal to $-I_{q}, I_{c}=0$, and liberation of hydrogen should entirely cease. If $-I$ be further increased in the negative direction, either metal will be dissolved or oxygen will be liberated in proportion to $-\left(I_{a}+I_{c}\right)$. If liberation of oxygen occurs, a discontinuity, even more abrupt than that due to liberation of hydrogen, may be observed.

The effect of any change in values of $r_{a}$ or $r_{c}$ may be interpreted from figure $3, A$. For example, if $\left(e_{a}-e_{c}\right)$ remains constant, and $r_{c}$ decreases, then $i_{o}$, according to eq 1 must increase. This will increase the numerical value of $-i_{o} r_{a}$, and, consequently, $E_{G}$ will be lowered. If, on the other hand, the cathodic resistance is increased by the presence of a hydrogen film, the accompanying change in resistive potential is in the same direction as an increase in the emf component, $e_{c}$, and $i_{o}$ must decrease. An increase in $r_{a}$ would result in a lowering of $E_{G}$ and conversely. It can therefore be seen that an increment or decrement in $E$ (also in $E_{G}$ ) may occur when $E>E_{a}$, or $E>E_{c}$, not only as the result of liberation of hydrogen, but also if the anodic or cathodic resistance changes.

\section{Multiple Galvanic Couples}

Let us now consider the electrical relations between two galvanic couples $(R)$ and $(O)$ having different potentials with reference to $E_{s}$. For example, in figure $4, E_{G R}$ may represent the reducing potential and $E_{G O}$, the oxidizing potential. Such conditions exist on iron in a wet clay where one area is poorly

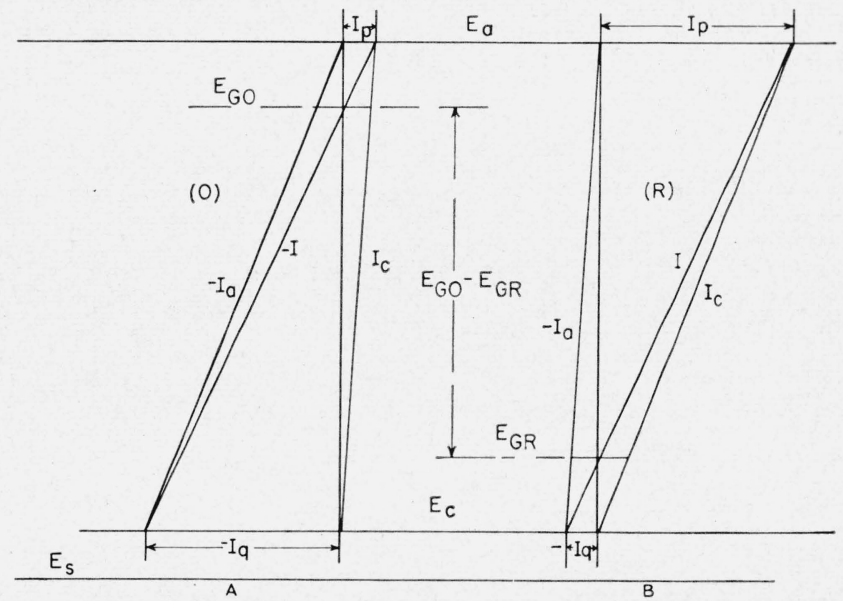

Figure 4. Multiple galvanic couples.

$A$, One at oxidizing potential, $E_{G o} ; \mathrm{B}$, one at reducing potential, $E_{G R}$.

aerated $(O)$ and the other is well aerated $(R)$. Then

where

$$
E_{G O}-E_{G R}=I^{\prime}\left(R_{G O}+R_{G R}+r\right),
$$

$$
\begin{aligned}
R_{G O}= & \text { the resistance of couple } E_{G O} . \\
R_{G R}= & \text { the resistance of couple } E_{G R} . \\
r= & \text { internal resistance of the path of } \\
& \text { current } I \text { between the couples (ex- } \\
& \text { ternal resistance being neglected). } \\
I^{\prime}= & \text { current supplied by the anodic couple } \\
& (O) \text { to the cathodic couple }(R) .
\end{aligned}
$$

Couple $(R)$ is therefore receiving some cathodic protection at the expense of couple $(O)$. Such protection is complete only if $I^{\prime}$ becomes equal to the $I_{p}$, required by the cathodic $(R)$ couple.

In this illustration, complete cathodic protection without external current is impossible because it would require the protective current $I_{p}$ to flow to the potential level $E_{a}$, which is higher than that of the current source. Such relations also apply in the field of metal coatings, where the coating may behave like a galvanic couple. If it is anodic toward the base metal, which itself also has a potential level depending on its galvanic-couple properties, then figure 4, shows that cathodic protection is not complete when the coating is perforated. For example, couple $(O)$ might represent a zinc coating on iron as the basis metal represented by $(R)$.

When an external potential difference $E_{\ell}$ is applied to multiple couples, the current distribution to them depends upon their $E_{G}$ values and the resistances $\underline{r}$ between them and the auxiliary electrode. Thus, in figure 5 , where a metal is represented as having a reducing $(R)$ and an oxidizing area $(O)$, local currents $i$ flow from the anodes $a$ to the cathodes $c$, in each area. In addition, a "long-line" current I' may flow from the local anodes in the oxidizing area to the local cathodes in the reducing area. If now $E_{t}$ be gradually increased from zero, current $\underline{i}_{C R}$ will begin to flow first to the area of lowest potential, that is, to 


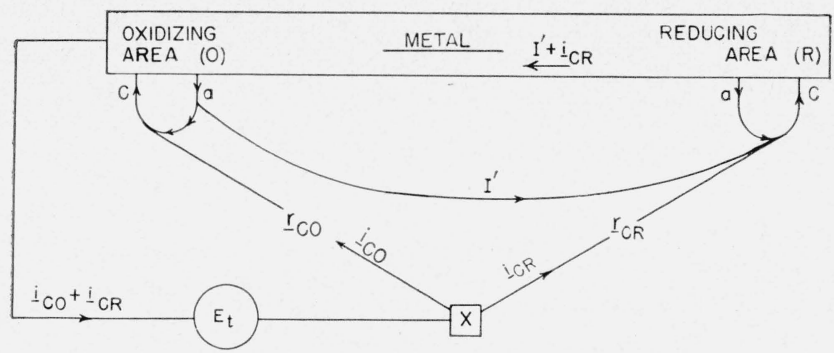

Figure 5. External potential difference, $E_{t}$, and current applied to multiple galvanic couples.

the cathodes of the reducing area. When

$$
E_{G R}+\underline{i}_{C R} \underline{r}_{C R}=E_{G O},
$$

$I^{\prime}$ becomes zero, and current $i_{C O}$ will begin flowing to the oxidizing area. Consequently, if $\underline{r}_{C R} \gg r_{C O}$, it may be possible to polarize the oxidizing area $(O)$ to the potential $E_{a}$, and achieve cathodic protection there, before it is possible at the reducing area, where a much higher value of $E_{t}$ may be required for complete cathodic protection. That is, even though the rate of corrosion may be less in the reducing area, a higher value of $E_{t}$ may be required there for complete protection, than in the oxidizing area. However, it is fortunate that protection in the oxidizing area, where it may be needed most, is the more easily accomplished.

Conversely, if $r_{C R} \ll r_{C O}$, a very high value of $i_{C R}$ may be required to meet the condition represented by eq 47. This is unfortunate, if corrosion is more severe in the oxidizing area.

\section{Summary}

The electrical theory of galvanic couples has been outlined as a basis for an understanding of the polarization of electrodes. It applies to most electrochemical processes including those in batteries, electrodeposition of metals and other electrolytic processes, and corrosion. By the technique of separated electrodes, some of the electrical quantities, which together comprise the characteristics of the galvanic couples, may be measured or computed. In the usual galvanic couple, which is a galvanic cell on closed circuit, electrical measurement is practically limited to determining its potential $E_{G}$ in reference to an electrode of constant potential $E_{s}$. The relations of $E_{G}$ to applied current $I$, and applied potential difference $E$, is developed for a couple having resistance $R_{G}$. From these, both "current" and "potential" criteria for cathodic protection are derived. The theory is also applied to multiple galvanic couples at different potentials.

\section{References}

[1] W. J. Müller, Korrosion u Metallschutz 13, 144 (1937). W. J. Müller, Trans. Electrochem. Soc. $\mathbf{7 6 ,} 167$ (1939). G. W. Akimow, Korrosion u Metallschutz 8, 201 (1932). G. W. Akimow and N. D. Tomaschow, Korrosion u Metallschutz 13, 114 (1937).

[2] R. H. Brown and R. B. Mears, Trans. Electrochem. Soc. $\mathbf{8 1}, 455$ (1942).

R. Landau and C. S. Oldach, Trans. Electrochem. Soc. 81, $521(1942)$.

R. Landau, Trans. Electrochem. Soc. 81, 559 (1942).

J. V. Petrocelli and A. Phillips, Trans. Electrochem. Soc. 85, 257 (1944).

[3] H. E. Haring and W. Blum, Trans. Electrochem. Soc. 44, $313(1923)$.

T. P. Hoar and J. N. Agar, Faraday Society, Electrode processes, Discussion No. 1, p. 162 (1947)

[4] R. B. Mears, J. Electrochem. Soc. 95, 1 (1949).

R. B. Mears and R. H. Brown, J. Electrochem. Soc. 97, 75 (1950).

[5] A. L. Ferguson, Trans. Electrochem. Soc. 76, 113 (1939).

[6] A. Hickling, Trans. Faraday Soc. 33, 1540 (1937).

[7] E. Newbury, et al., Trans. Faraday Soc. 43, 123 (1947) E. Newbury, Trans. Faraday Soc. 43, 127 (1947).

[8] H. D. Holler, J. Electrochem. Soc. 97, 271 (1950).

Washington, June 1, 1950. 